\title{
DESARROLLO DE VARIEDADES TROPICALES DE SOYA PARA EL CONSUMO HUMANO 1
}

\author{
Enrique Villalobos ${ }^{2}$, Fausto Camacho ${ }^{2}$
}

\begin{abstract}
RESUMEN
Desarrollo de variedades tropicales de soya para el consumo humano. Se desarrollaron líneas de soya (Glycine $\max$ L. Merr.) con semillas sin las lipoxigenasas 2 y 3 y con adaptación a condiciones tropicales fueron desarrolladas por el Centro de Investigaciones en Granos y Semillas, de la Universidad de Costa Rica. Estas líneas se obtuvieron del cruce de plantas $\mathrm{F}_{3}$ seleccionadas del cruce de 'Kanto-101' $\mathrm{X}$ 'IAC8 ' con plantas $\mathrm{F}_{2}$ seleccionadas del cruce de 'Padre' $\mathrm{X}$ 'Duocrop'. Kanto-101 es una variedad japonesa que se usó como donadora de los genes $\operatorname{lox}_{2}$ y $\operatorname{lox}_{3}$, que inhiben la síntesis de las lipoxigenasas 2 y 3 en la semilla de soya. La eliminación de las lipoxigenasas 2 y 3 mejora considerablemente las características sensoriales de la leche de soya. La variedad $\mathrm{Pa}-$ dre, se usó como donadora de los genes que retardan la floración en condiciones de día corto. Las líneas $\mathrm{F}_{11}$ CIGRAS-34, 47,51 y $57\left(\operatorname{lox}_{2}, \operatorname{lox}_{3}\right)$ derivadas del cruzamiento doble, mostraron un buen comportamiento agronómico cuando se compararon en tres localidades con las variedades CIGRAS-06 y 10 (Lox), previamente liberadas por su alta productividad. El uso del cultivar Padre, portador de los genes que retardan la floración en condiciones de día corto, en los programas de mejoramiento genético de la soya en el trópico, mostró su gran potencial al generar materiales tardíos y con buena adaptación. Los materiales sin lipoxigenasas en la semilla alcanzan un mejor precio en el mercado internacional y son una buena alternativa para la exportación a países donde la soya es parte importante de la dieta humana.
\end{abstract}

\begin{abstract}
Breeding tropical soybeans for human consumption. Soybean (Glycine $\max$ L. Merr.) lines lacking seed lipoxigenases 2 and 3 and with high yield potential were developed at the Grain and Seed Research Center of the University of Costa Rica. Those lines were obtained by crossing selected $\mathrm{F}_{3}$ plants from 'Kanto-101' $\mathrm{X}$ 'IAC-8' with $F_{2}$ plants selected from 'Padre' $X$ 'Duocrop'. Kanto-101 is a Japanese variety that was used as a donor of $\operatorname{lox}_{2}$ and $\operatorname{lox}_{3}$ genes that inhibit the synthesis of lipoxigenases 2 and 3 in the soybean seed. Elimination of lipoxigenases 2 and 3 considerably improves the taste and odor of the soybean milk. Cultivar Padre was used as a donor of delayed flowering under short day conditions. $\mathrm{F}_{11}$ lines CIGRAS-34, 47,51 and $57\left(\operatorname{lox}_{2}, \operatorname{lox}_{3}\right)$, derived from the double cross, showed an outstanding agronomic performance when compared with high yielding cultivars CIGRAS-06 and 10 (Lox), in three sites. The use of Padre cultivar, carrier of the genes that delay flowering under short day conditions, in tropical soybean breeding programs, is important to develop late maturing lines with good adaptation in tropical regions. Lipoxigenase-free seed of soybeans reach a higher price in the international market and might be a potential export crop to those countries where soybeans are an important component of the human diet.
\end{abstract}

\section{INTRODUCCIÓN}

El uso de la soya en la dieta regular de la población en el trópico americano, es muy baja. Sin embargo, en los últimos años, el interés por el uso de la soya para el consumo humano ha venido en ascenso, i) por el mejor conocimiento que la población ha adquirido sobre el alto valor nutricional y medicinal de esta leguminosa $\mathrm{y}$ ii) por la introducción en nuestros países de alimentos palatables y que emplean la soya como ingrediente importante o principal, como es el caso de las bebidas que se preparan con "leche" de soya y frutas, o las tortas de carne de las hamburguesas.

El sabor a frijol de la semilla de soya es uno de los factores que limitan el consumo humano de esta legu-

\footnotetext{
1 Parte del proyecto VI-734-97-327 Vicerrectoría de Investigación. Universidad de Costa Rica.

2 Centro de Investigaciones en Granos y Semillas (CIGRAS), Ciudad Universitaria Rodrigo Facio, Universidad de Costa Rica. San Pedro, San José, Costa Rica. E-mail: enriquev@cariari.ucr.ac.cr
} 
minosa. Este sabor indeseable es producido por tres isoenzimas lipoxigenasas que son codificadas por tres alelos dominantes (Lox) (Hildebrand, 1989). Las lipoxigenasas catalizan la hidroperoxidación de los ácidos grasos linoleico y linolénico y producen cantidades muy bajas (aprox. 5 ppb) del aldehído hexanal, pero suficientes para producir ese sabor y olor desagradables de la semilla (Hildebrand, 1989). La isoenzima 3 es la más abundante, pero la 2 , aunque es la menos abundante, exhibe la mayor actividad específica, por lo que, en términos de actividad, las tres isoenzimas están presentes en proporciones similares (Kitamura, 1984). Wilson (1996) ha presentado evidencia de que la simple eliminación de la lipoxigenasa 2 es suficiente para mejorar, en forma considerable, el sabor y el olor de la leche de soya y sus derivados. Actualmente existen cultivares $\left(\operatorname{lox}_{1}, \operatorname{lox}_{3}\right),\left(\operatorname{lox}_{2}, \operatorname{lox}_{3}\right)$ y $\left(\operatorname{lox}_{1}, \operatorname{lox}_{2}, \operatorname{lox}_{3}\right)$, pero el desarrollo de los genotipos $\left(\operatorname{lox}_{1}, \operatorname{lox}_{2}\right)$ y de los triple recesivos ha sido más difícil, debido a que los loci $\operatorname{lox}_{1}$ y lox 2 están estrechamente ligados (Hildebrand, 1989; Wilson, 1996). No se encontró información sobre el desarrollado de cultivares de soya sin lipoxigenasas en el trópico.

Tomando en consideración estos antecedentes, el Centro de Investigaciones en Granos y Semillas (CIGRAS), decidió incluir como un objetivo importante de su programa de mejoramiento genético de la soya, el desarrollo de cultivares con mejores características sen- soriales que las variedades corrientes, mediante la eliminación de las lipoxigenasas 2 y 3 de sus semillas. Como los genes lox están presentes en unos pocos cultivares de soya que se ubican en los grupos de madurez temprana y por lo tanto de difícil adaptación al trópico, para desarrollar variedades $\operatorname{lox}_{2}, \operatorname{lox}_{3}, y$ con buena adaptación en estas condiciones, se empleó el cultivar 'Padre' (Hartwig et al., 1988), poseedor de los "genes de floración retardada en condiciones de día corto" (Hartwig y Kiihl, 1979). El cruzamiento del cultivar Padre con otros materiales sub-tropicales o pertenecientes a los grupos de madurez tardía, generó una gran variabilidad genética (Villalobos y Camacho, 1999). Esto favorece, obviamente, el proceso de selección de materiales sobresalientes y con buena capacidad de adaptación en el trópico.

\section{MATERIALES Y MÉTODOS}

El cruzamiento de plantas seleccionadas $\mathrm{F}_{3}$, carentes de las lipoxigenasas 2 y 3 en sus semillas y provenientes del cruce de 'IAC-8' X 'Kanto-101', con plantas $\mathrm{F}_{2}$ sobresalientes, producto del cruce de 'Padre' $\mathrm{X}$ 'Duocrop', produjo varias líneas sin lipoxigenasas 2 y 3 en sus semillas, por poseer los genes $\operatorname{lox}_{2}$ y $\operatorname{lox}_{3} y$ lo suficientemente tardías para dar una buena producción de semilla, por poseer los genes de floración retardada en condiciones de día corto (Figura 1). Además, de proveer

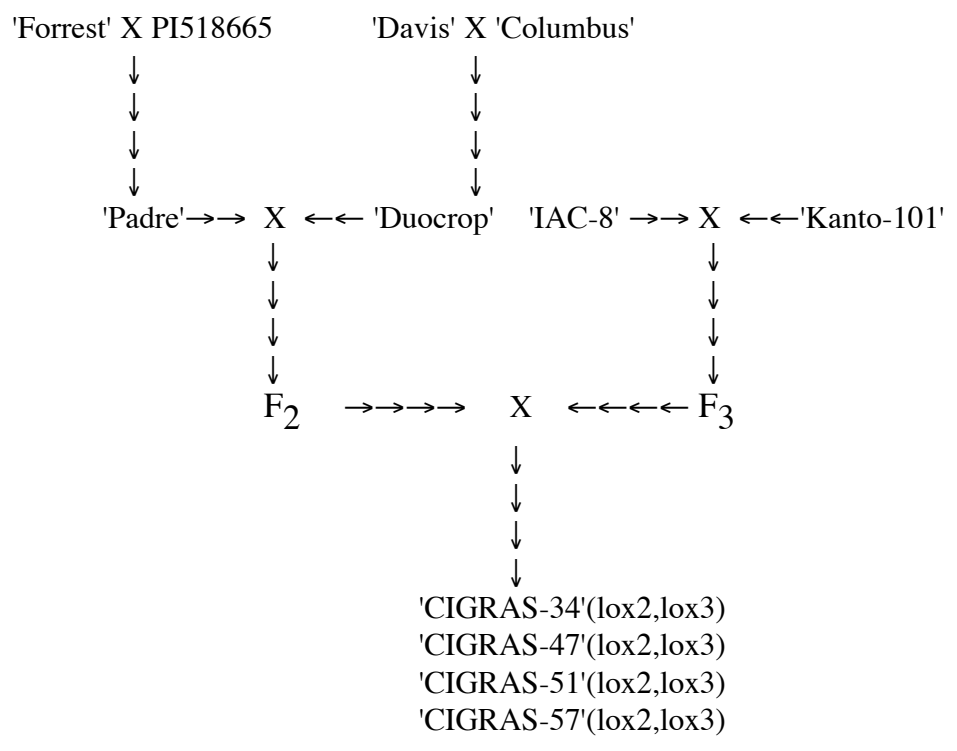

Figura 1. Origen de las variedades CIGRAS-34, 47, 51,57 y 63 que combina los genes de floración retardada en condiciones de día corto, provenientes de la variedad Padre y los genes $\operatorname{lox}_{2}$ y $\operatorname{lox}_{3}$, que inhiben la síntesis de las lipoxigenasas 2 y 3 en la semilla, respectivamente, y provenientes de la variedad Kanto-101. 
los genes $\operatorname{lox}_{2}$ y $\operatorname{lox}_{3}$ la variedad Kanto-101 posee resistencia a varias razas del virus del mosaico común (Camacho, 1989). La variedad 'IAC-8' es de origen brasileño y se cultivó ampliamente en Costa Rica y en algunos países de América Central en los años 80. La variedad 'Padre' es producto del cruce de 'Forrest' $\mathrm{X}$ D77-12480 (Hartwig et al., 1988) y al poseer el carácter de floración retardada en condiciones de día corto, que se debe a la presencia de 3 o más genes (Hartwig y Kiihl, 1979), se adapta relativamente bien en condiciones de días cortos. En Costa Rica, contrario a lo que ocurrió con otras variedades del grupo de madurez VII, Padre mostró un comportamiento aceptable, en cuanto a madurez y producción, aunque alcanzó un grado relativamente alto de volcamiento. Duocrop también se ubica en el grupo de madurez VII y se originó del cruce de 'Davis' X 'Columbus' y contrario a Padre, su adaptación en Costa Rica y en otros países tropicales fue muy pobre, debido a su precocidad (Jackobs, et al., 1984).

Las poblaciones segregantes del cruzamiento original, hasta la $\mathrm{F}_{5}$, se manejaron como un lote ("bulk"), eliminando aquellas plantas muy precoces y las muy tardías. Plantas $\mathrm{F}_{6}$ obtenidas de semillas con genotipo $\operatorname{lox}_{2}, \operatorname{lox}_{3}$ previamente comprobado y con apariencia y producción sobresaliente, se sembraron en surcos individuales. La semilla de los surcos sobresalientes se mezcló y se sembró en las siguientes localidades: Estación Experimental Fabio Baudrit en Alajuela, a $10^{\circ} 05^{\prime}$ $\mathrm{N}$ y $840 \mathrm{msnm}$, Liberia $10^{\circ} 27^{\prime} \mathrm{N}$ y $60 \mathrm{msnm}$ y San Carlos $10^{\circ} 28^{\prime} \mathrm{N}$ y 250 msnm, para selección visual. En la Azucarera El Viejo, Carrillo, Guanacaste a $10^{\circ} 26^{\prime} \mathrm{N}$ y a 17 msnm, de enero a abril de 1998, bajo irrigación por gravedad; Los Chiles, Alajuela, a $11^{\circ} \mathrm{N}$ y $30 \mathrm{msnm}$ y en Upala, Alajuela, a $10^{\circ} 54^{\prime} \mathrm{N}$ y $50 \mathrm{msnm}$, de diciembre a abril de 1999, en pruebas comparativas siguiendo la metodología del Programa Internacional de Soya de la Universidad de Illinois (Jackobs et al., 1984). Las unidades experimentales consistieron de 5 surcos de 5 metros de longitud, separados entre sí a 50 $\mathrm{cm}$, en un diseño experimental de bloques completos al azar con cuatro repeticiones. Al momento de la cosecha, se eliminaron los dos surcos externos y los $50 \mathrm{~cm}$ de los extremos de los tres surcos centrales. Se usó una densidad de población que varió entre 10 y 12 plantas por metro lineal (200.000 a 240.000 plantas por hectárea). Se evaluaron las siguientes variables: producción de semilla ( $12 \%$ de humedad); el peso de 100 semillas; la altura de la planta y de la inserción de las primeras vainas; el acame en una escala visual de uno a cinco, donde el valor inferior corresponde a una posición vertical de las plantas y el último a una posición totalmente horizontal; los días a floración $\left(\mathrm{R}_{2}\right)$ y a la madurez fisiológica $\left(R_{7)}\right.$ y el número de nudos correspondiente a estos dos estados del desarrollo de la planta.
Las variedades que se evaluaron en esta investigación fueron: CIGRAS-34, 47,51 y 57 que carecen de las lipoxigenasas dos y tres en sus semillas y junto con CIGRAS-63, que solamente carece de la lipoxigenasa 2, se derivan del cruzamiento doble que se presenta en la Figura 1. CIGRAS-06,10 y 17 fueron seleccionadas del cruzamiento de Padre X Duocrop y poseen las tres lipoxigenasas en sus semillas. CIGRAS-06 se empleó como testigo, dada su alta productividad en evaluaciones anteriores en Costa Rica (Villalobos y Camacho, 1999; Villalobos y Camacho, en prensa) y Nicaragua (Busti1lo, 1999). El cultivar IAC-8 se usó también como testigo, por haber sido ampliamente cultivado en Costa Rica y otros países centroamericanos en los años 80 (Montero, 1987).

Para evaluar la interacción genotipo x localidad para todas las variables que se evaluaron, se hizo un análisis de varianza combinando los datos de las tres localidades y empleando la localidad como la parcela principal y al genotipo como la sub-parcela. Además, se hizo un análisis de varianza para cada localidad, con el fin de comparar los genotipos en la producción de semilla, el peso de 100 semillas y el acame en cada localidad.

\section{Análisis de las lipoxigenasas}

El procedimiento para detectar la presencia de lipoxigenasas en la semilla de soya, consistió en evaluar, colorimétricamente el tiempo de decoloración de los $\beta$ carotenos extraídos de la zanahoria cruda. Diez $\mathrm{ml}$ de un extracto crudo de b-carotenos en acetona se diluyen con $90 \mathrm{ml}$ de agua destilada hasta lograr una solución con 0,85 de absorbancia. En un vial de cinco ml se depositan dos ml de la solución diluida de carotenoides y aproximadamente $10 \mathrm{mg}$ de semilla de soya. Se agita el vial durante unos 20 segundos y se deja reposar. Esta solución se decolora más o menos rápido, según el tipo de lipoxigenasas presentes en la semilla así: $\left(\operatorname{Lox}_{1}\right.$, $\left.\operatorname{Lox}_{2}, \operatorname{Lox}_{3}\right)$ cinco minutos; $\left(\operatorname{Lox}_{1}, \operatorname{Lox}_{2}\right)$ y $\left(\operatorname{Lox}_{2}\right) 15$ minutos; $\left(\operatorname{Lox}_{1}, \operatorname{Lox}_{3}\right) 1$ hora y $\left(\operatorname{Lox}_{1}\right) 12$ horas (Camacho, 1994).

\section{RESULTADOS Y DISCUSIÓN}

Los países de latitudes extremas tienen la ventaja sobre los países que se ubican en el trópico de cáncer, en que éstos pueden hacer un mejor aprovechamiento de los cultivares mejorados de soya que se generan en las regiones que se ubican en la misma latitud del hemisferio opuesto, mediante la simple introducción y reproducción de los mismos. En el trópico, los programas de mejoramiento genético de la soya son relativamente 
escasos y la introducción de variedades mejoradas, aún de aquellas que se ubican en los grupos de madurez tardía (Sur de los Estados Unidos o Norte de Brasil), no han dado buenos resultados, por el comportamiento precoz de estos materiales, cuando se cultivan en condiciones de días cortos.

Los resultados de esta investigación, así como aquellos obtenidos anteriormente (Villalobos y Camacho, 1999), muestran que la inclusión del cultivar Padre, y más específicamente, el aprovechamiento de los genes que retardan la floración en condiciones de días cortos (Hartwig y Kiihl, 1979) en los programas de mejoramiento genético de la soya en los trópicos, es una excelente opción para obtener materiales de soya con una productividad alta en estas condiciones. El cruzamiento de Padre con varios de los materiales de soya de la colección del CIGRAS, generó una variabilidad genética muy amplia en la generación $\mathrm{F}_{2}$. En el número de días a la floración, se obtuvo plantas $\mathrm{F}_{2}$ que florecieron a los 29 días y otras que alcanzaron este estado de desarrollo a los 90 días, (Villalobos y Camacho, 1999; Villalobos y Camacho, en prensa), aspecto totalmente inesperado e inusual en condiciones tropicales, y que permite obviamente, seleccionar aquellos materiales que mejor se adapten a estas condiciones. Más aún, el empleo del cultivar Padre en los programas de mejoramiento genético en el trópico, facilita el aprovechamiento de características especiales y que están presentes en cultivares pertenecientes a los grupos de madurez temprana y por lo tanto inadaptables en el trópico por su precocidad, como es el caso que nos ocupa de los genes lox, presentes en el cultivar Kanto-101. El cultivar Kanto-101 florece en Costa Rica en menos de 30 días y su desarrollo y su potencial de producción son sumamente pobres, pero su valiosa contribución como donador de los genes lox pudo lograrse mediante la combinación de estos genes con aquellos presentes en el cultivar Padre, que favorecen un mayor desarrollo de la planta y una mayor producción de semilla, al retardar la floración en nuestras condiciones fotoperiódicas. El cruzamiento de Padre X Duocrop fue el que generó el material segregante más promisorio y que a la postre permitió seleccionar la variedad CIGRAS-06, que ha mostrado un comportamiento muy bueno en Costa Rica (Villalobos y Camacho, 1999; Villalobos y Camacho, en prensa) y en Nicaragua (Bustillo, 1999); de nuevo, Duocrop, es una variedad precoz y de baja productividad en el trópico (Jackobs et al., 1984).

\section{Propiedades sensoriales de las variedades $\operatorname{lox}_{2}, \operatorname{lox}_{3}$}

Las líneas avanzadas $\operatorname{lox}_{2} \operatorname{lox}_{3}$ que se evaluaron en esta investigación, carecen del olor y sabor a frijol, típico de la semilla de soya. Muchos trabajos (Wilson,
1996) han demostrado que la "leche" de soya y sus derivados, producidos con semilla que carece de la lipoxigenasa dos únicamente, tienen mejor sabor y olor que aquellos producidos con soya corriente. El olor y sabor desagradables de la semilla de soya pueden eliminarse con el calentamiento, sin embargo, las temperaturas altas tienen un efecto negativo en la calidad nutricional de los productos que se derivan de la leche de soya, en tanto que desnaturalizan otras proteínas además de las lipoxigenasas (Wilson, 1996). La ventaja de la leche producida con variedades $\operatorname{lox}_{2}, \operatorname{lox}_{3}$, es que no requieren mucho calentamiento para dar un producto de buen sabor, de manera que otras proteínas no se desnaturalizan. Estos genotipos han sido objeto de trabajos experimentales orientados al uso de la leche de soya y otros productos en la alimentación humana en la Universidad de Costa Rica, con resultados positivos (datos sin publicar).

La producción de variedades de soya sin lipoxigenasas en el trópico, no solamente representa una alternativa para el consumo humano, sino que también es una buena alternativa para la exportación a aquellos países donde la soya es parte regular de la dieta humana y donde se paga un precio más alto por estos materiales (Wilson, 1996).

\section{La interacción genotipo x localidad}

En varias evaluaciones de materiales de soya CIGRAS que se hicieron en varias localidades de Costa Rica en años anteriores a 1998, y bajo condiciones favorables de humedad en el suelo o bajo irrigación, se logró confirmar que no hubo interacción genotipo x localidad, como sí ocurrió en esta oportunidad para la producción de semilla y otras características (Cuadro 1). Esas evaluaciones mostraron que las variedades CIGRAS-06 y CIGRAS-10, ocuparon el primer lugar en producción de semilla, consistentemente (Villalobos y Camacho, 1999). Esa tendencia se observó en el presente trabajo, cuando las nueve variedades bajo evaluación se compararon en Carrillo, bajo riego, y en Upala, cuando no hubo limitaciones de humedad en el suelo (Cuadro 2). No obstante, cuando la evaluación de los materiales se hizo en Los Chiles, donde hubo déficit hídrico por un período de tres semanas antes de la cosecha, aquellos materiales más tardíos, como CIGRAS06, 10 y 17, fueron más seriamente afectados por el estrés hídrico, que redujo, principalmente, el peso de la semilla (Cuadro 2). Por otra parte, las demás variedades, que pudieron cosecharse unos 10 días antes que las anteriores, fueron lógicamente, menos afectadas en el peso de la semilla y en la producción que sus contrapartes Lox (Cuadros 1 y 2). En Los Chiles los materiales no manifestaron ningún grado de volcamiento, que es un aspecto que afecta seriamente la producción de semilla 
Cuadro 1. Análisis de varianza para algunas características agronómicas y fenológicas de nueve variedades de soya, combinando tres localidades: Carrillo (1998), Upala y Los Chiles (1999).

\begin{tabular}{|c|c|c|c|c|c|c|c|c|c|}
\hline $\begin{array}{c}\text { Línea o Variedad } \\
\text { (Genotipo) }\end{array}$ & $\begin{array}{l}\text { Producción } \\
\text { (kg/ha) }\end{array}$ & $\begin{array}{c}\text { Peso }(\mathrm{g}) \\
100 \\
\text { semillas }\end{array}$ & $\begin{array}{c}\text { Altura } \\
\text { planta } \\
(\mathbf{c m})\end{array}$ & $\begin{array}{c}\text { Altura } \\
\text { vainas } \\
(\mathrm{cm})\end{array}$ & $\begin{array}{c}\text { Acame } \\
(1-5)\end{array}$ & $\begin{array}{c}\text { Días } \\
\mathbf{R}_{2}\end{array}$ & $\begin{array}{l}\text { Días } \\
\mathbf{R}_{7}\end{array}$ & $\begin{array}{c}\text { Nudos } \\
\mathbf{R}_{2}\end{array}$ & $\begin{array}{c}\text { Nudos } \\
\mathbf{R}_{7}\end{array}$ \\
\hline $\begin{array}{l}\text { IAC-08 } \\
\text { (Lox 1,Lox2,Lox3) }\end{array}$ & 3171 & 20,5 & 62 & 17 & 2,0 & 40 & 100 & 9,5 & 11,1 \\
\hline $\begin{array}{l}\text { CIGRAS-06 } \\
\text { (Lox } 1, \text { Lox } 2, \text { Lox } 3)\end{array}$ & 3460 & 17,3 & 102 & 23 & 1,2 & 47 & 110 & 11,0 & 16,4 \\
\hline $\begin{array}{l}\text { CIGRAS-10 } \\
\text { (Lox } 1, \text { Lox } 2, \text { Lox } 3)\end{array}$ & 3404 & 18,0 & 95 & 22 & 1,2 & 48 & 110 & 11,3 & 16,6 \\
\hline $\begin{array}{l}\text { CIGRAS-17 } \\
\text { (Lox } 1, \operatorname{Lox} 2, \operatorname{Lox} 3)\end{array}$ & 3296 & 17,8 & 98 & 22 & 1,3 & 48 & 110 & 11,8 & 17,6 \\
\hline $\begin{array}{l}\text { CIGRAS-34 } \\
\text { (Lox } 1, \operatorname{lox} 2, \operatorname{lox} 3)\end{array}$ & 3330 & 17,7 & 76 & 19 & 1,2 & 43 & 100 & 12,1 & 14,9 \\
\hline $\begin{array}{l}\text { CIGRAS-47 } \\
\text { (Lox } 1, \text { lox } 2, \operatorname{lox} 3)\end{array}$ & 3103 & 17,2 & 76 & 18 & 1,4 & 45 & 102 & 12,1 & 16,6 \\
\hline $\begin{array}{l}\text { CIGRAS-51 } \\
\text { (Lox } 1, \text { lox } 2, \text { lox3) }\end{array}$ & 3327 & 17,8 & 87 & 21 & 1,3 & 46 & 101 & 11,5 & 17,5 \\
\hline $\begin{array}{l}\text { CIGRAS-57 } \\
\text { (Lox } 1, \operatorname{lox} 2, \operatorname{lox} 3)\end{array}$ & 3275 & 17,3 & 87 & 20 & 1,6 & 46 & 102 & 11,9 & 16,8 \\
\hline $\begin{array}{l}\text { CIGRAS-63 } \\
\text { (Lox1,Lox2,lox3) }\end{array}$ & 3133 & 18,1 & 79 & 21 & 1,5 & 42 & 100 & 11,0 & 15,3 \\
\hline ANDEVA & & & & & & & & & \\
\hline Localidad (L) & $*$ & $* *$ & $* *$ & $* *$ & $* *$ & $* *$ & $* *$ & $* *$ & $* *$ \\
\hline Genotipo (G) & ns & ns & $* *$ & $\mathrm{~ns}$ & ns & $* *$ & $* *$ & $* *$ & $* *$ \\
\hline GX L & $* *$ & ns & $* *$ & $* *$ & $*$ & $* *$ & ns & $* *$ & ns \\
\hline
\end{tabular}

ns; *; **; no significativo y significativo al nivel de $\mathrm{p} \leq 0,05$ y $\mathrm{p} \leq 0,001$ respectivamente.

(Specht, et al., 1999), y que favoreció también a aquellos cultivares como IAC-8, que bajo condiciones de disponibilidad de agua adecuada, muestran un grado de volcamiento alto, a pesar de su precocidad (Cuadro 2).

\section{Adaptación de las variedades lox}

Si bien es cierto que las variedades lox incluidas en este experimento no logran superar a la variedad CI-
GRAS-06, que al presente es la variedad más productiva en Costa Rica, logran alcanzar una producción alta en condiciones ambientales contrastantes y por lo tanto, son recomendables para su explotación comercial en Costa Rica, especialmente si se toma en consideración que éstos podrían ser materiales con un potencial alto para la exportación y con un mejor precio en el mercado internacional (Wilson, 1996).

Cuadro 2. Valores promedio y análisis de varianza de la producción de semilla por área, del peso de 100 semillas y del volcamiento de nueve variedades de soya en Carrillo (1998), Upala y Los Chiles (1999).

\begin{tabular}{|c|c|c|c|c|c|c|c|c|c|}
\hline \multirow[t]{2}{*}{ Variedad } & \multicolumn{3}{|c|}{ Carrillo } & \multicolumn{3}{|c|}{ Upala } & \multicolumn{3}{|c|}{ Los Chiles } \\
\hline & $\begin{array}{c}\text { Producción } \\
\text { (kg/ha) }\end{array}$ & $\begin{array}{c}\text { Peso } 100 \\
\text { semillas (g) }\end{array}$ & $\begin{array}{l}\text { Acame } \\
(1-5)\end{array}$ & $\begin{array}{c}\text { Producción } \\
\text { (kg/ha) }\end{array}$ & $\begin{array}{c}\text { Peso } 100 \\
\text { semillas (g) }\end{array}$ & $\begin{array}{c}\text { Acame } \\
(1-5)\end{array}$ & $\begin{array}{l}\text { Producción } \\
\text { (kg/ha) }\end{array}$ & $\begin{array}{c}\text { Peso } 100 \\
\text { semillas (g) }\end{array}$ & $\begin{array}{l}\text { Acame } \\
(1-5)\end{array}$ \\
\hline IAC-8 & 2985 & 20,5 & 2,2 & 2971 & 23,2 & 3,0 & 3551 & 18,3 & 1,0 \\
\hline CIGRAS-06 & 3532 & 17,5 & 1,7 & 4212 & 20,7 & 1,3 & 2863 & 13,8 & 1,0 \\
\hline CIGRAS-10 & 3090 & 18,0 & 1,3 & 4362 & 20,6 & 1,5 & 3036 & 13,3 & 1,0 \\
\hline CIGRAS-17 & 3117 & 18,5 & 1,0 & 3968 & 20,3 & 1,9 & 2791 & 13,1 & 1,0 \\
\hline CIGRAS-34 & 3070 & 17,5 & 1,0 & 3270 & 19,6 & 1,5 & 3643 & 15,9 & 1,0 \\
\hline CIGRAS-51 & 2945 & 16,6 & 1,0 & 3223 & 18,9 & 2,2 & 3120 & 15,7 & 1,0 \\
\hline CIGRAS-57 & 3343 & 18,0 & 1,0 & 3625 & 19,6 & 2,2 & 3119 & 15,2 & 1,0 \\
\hline CIGRAS-47 & 3373 & 17,7 & 1,0 & 3423 & 19,9 & 2,8 & 3059 & 14,2 & 1,0 \\
\hline CIGRAS-63 & 2900 & 17,5 & 1,3 & 3084 & 20,7 & 2,2 & 3360 & 16,2 & 1,0 \\
\hline \multicolumn{10}{|l|}{ ANDEVA } \\
\hline Variedades & $*$ & $* *$ & $* *$ & $*$ & $* *$ & $*$ & $*$ & $* *$ & ns \\
\hline DMS $(a \leq 0,05)$ & 480 & 0,08 & 0,6 & 816 & 1,7 & 1,0 & 582 & 1,0 & 0,0 \\
\hline
\end{tabular}

ns; *; **; no significativo y significativo al nivel de $p \leq 0,05$ y $p \leq 0,001$ respectivamente. 
Cabe resaltar el comportamiento de la variedad CIGRAS-34, que en Los Chiles logró superar incluso a la variedad CIGRAS-06, debido a su precocidad que le permitió escapar a la sequía (Cuadro 2). Esta localidad, que presenta una precipitación muy alta y que prácticamente impide que se pueda sembrar la soya antes del mes de diciembre, también es afectada por el déficit hídrico en marzo y abril. Esto señala que las variedades de soya que podrían adaptarse bien en esta situación particular deben ser lo suficientemente precoces para escapar del déficit hídrico. La variedad CIGRAS-34, además de su relativa precocidad, presenta una característica muy importante y es la resistencia al deterioro de la semilla en condiciones adversas de almacenamiento (Zamora, 2000), lo cual le adiciona una ventaja comparativa sobre los demás materiales CIGRAS, y la posible recomendación para su explotación comercial en esta zona.

\section{AGRADECIMIENTOS}

Los autores desean dejar patente su agradecimiento post-mortem con el Dr. Edgar Hartwig, de la Universidad de Mississippi, por el suministro de la semilla del cultivar Padre y sus valiosos consejos para la ejecución de este trabajo. El Dr. Hartwig, quien murió en 1996, hizo una enorme contribución en el manejo y el mejoramiento genético de la soya y no escatimó esfuerzos para colaborar con los programas de mejoramiento genético de la soya en el trópico. Junto con los Drs. Hinson (creador de la variedad Júpiter) y Scott, el Dr. Hartwig desarrolló la variedad Padre. También queremos agradecer al MSc. Fermín Subirós, al Sr. Steven Polen y al Sr. Levi Friessen, su colaboración en la atención de las parcelas experimentales, y al MSc. Oscar Acuña, por facilitarnos el uso de una trilladora para cosechar la soya.

\section{LITERATURA CITADA}

BUSTILLO, C. 1999. Informe sobre los resultados de una prueba de variedades nuevas de soya. Ministerio Agropecuario y Forestal, Centro Experimental de Occidente, Managua, Nicaragua. Informe Técnico, 15p.

CAMACHO, F. 1989. Características y uso potencial para frijol de soja verde (edamame) de las variedades de soja nuevas y antiguas de la parte central de Japón. Informe Técnico. Nagano Chusin Agricultural Experiment Station, Shiojiri, Nagano, Japón. 15p.
CAMACHO, F. 1994. Características agronómicas y determinación de lipoxigenasas en una población segregante de soya adaptada al trópico. Tesis de MSc. en Ciencias en Agronomía, Colegio de Ciencias Agrícolas, Universidad de Puerto Rico, Mayaguez, Puerto Rico. 98p.

HARTWIG, E.E.; HINSON, K.; SCOTT, A. 1988. Registration of 'Padre' soybean. Crop Sci. 28:1025.

HARTWIG, E.E.; HIIHL, R. A. 1979. Identification and utilization of a delayed flowering character in soybeans for short-day conditions. Field Crops Res. 2:145-151.

HILDEBRAND, D.F. 1989. Lipoxygenases. Physiol. Plant. 76:249-253.

JACKOBS, J.; SMITH, C.; ERICKSON, D. 1984. International soybean variety experiment. XI Report of Results, International Soybean Program Series No. 29. College of Agriculture, University of Illinois, Urbana-Champaign, Illinois. $168 \mathrm{p}$.

MONTERO, R. 1987. Variedad IAC-8 de soya (Glycine max L. Merr.): una alternativa para el litoral pacífico de Costa Rica. Agron. Costarr. 12:107-111.

KITAMURA, K. 1984. Biochemical characterization of lipoxygenase-lacking mutants, L-1 less, L-2 less and L3 less soybeans. J. Agric. Biol. Chem. 48: 2339-2343.

SPETCH, J.E., HUME, D.J.; KUMUDINI, S.V. 1999. Soybean yield potential. A genetic and physiological perspective. Crop Sci. 39:1560-1570.

VILLALOBOS, E.; CAMACHO, F. 2000. Registration of CIGRAS.06 soybean. Crop Sci. (enviado para publicación).

VILLALOBOS, E.; CAMACHO, F. 1999. Avances en el mejoramiento genético de la soya en Costa Rica. II. CIGRAS-06 y CIGRAS-10, dos nuevas variedades tropicales. Agron. Costarr. 23:61-67.

WILSON, L.A. 1996. Comparison of lipoxygenase-null and lipoxygenase-containing soybeans for foods. In: Lipoxygenase and lipoxygenase pathway enzymes. Ed. By G.J. Piazza. AOCS Press, Champaign, Illinois, USA. pp.209-226.

ZAMORA, J. 2000. Germinación y vigor de la semilla de soya (Glycine $\max$ L. Merr.) sin algunas lipoxigenasas, en condiciones tropicales adversas. Tesis de Licenciatura, Escuela de Fitotecnia, Universidad de Costa Rica. 30 p. 